\title{
DETECTION OF EXTENDED SPECTRUM BETA LACTAMASES, AMPC BETA LACTAMASES AND METALLOBETALACTAMASES IN CLINICAL ISOLATES OF CEFTAZIDIME RESISTANT PSEUDOMONAS AERUGINOSA
}

\author{
Sivaraman Umadevi*, Noyal M Joseph, Kandha Kumari, Joshy M Easow, Shailesh Kumar, Selvaraj Stephen, Sreenivasan \\ Srirangaraj, Sruthi Raj
}

Department of Microbiology, Mahatma Gandhi Medical College and Research Institute, Pondicherry, India.

Submitted: June 14, 2010; Returned to authors for corrections: April 06, 2011; Approved: June 06, 2011.

\begin{abstract}
We studied the prevalence of ceftazidime resistance in Pseudomonas aeruginosa and the rates of extendedspectrum $\beta$-lactamase (ESBL), AmpC $\beta$-lactamase (AmpC) and metallo- $\beta$-lactamase (MBL) production among the ceftazidime resistant Pseudomonas aeruginosa. A very high rate of MBL production was observed, which suggested it to be an important contributing factor for ceftazidime resistance among Pseudomonas aeruginosa.
\end{abstract}

Key words: Extended-spectrum $\beta$-lactamases, AmpC, metallo- $\beta$-lactamases, ceftazidime resistance, Pseudomonas aeruginosa

Nosocomial infections caused by $P$. aeruginosa are frequently life threatening and often challenging to treat $(6,10)$. P. aeruginosa has an inducible, naturally occurring cephalosporinase that confers low-level resistance to aminopenicillins and narrow-spectrum cephalosporins such as cephalothin and cefoxitin (4,7). Emergence of resistance to multiple drugs due to selection of resistance during antipseudomonal therapy among the initially susceptible isolates occurs frequently with this pathogen, as it is more efficient in acquiring drug resistance by various mechanisms (1).

Resistance to extended spectrum cephalosporins may arise from over expression of the naturally occurring cephalosporinase or acquired beta-lactamases such as extended-spectrum $\beta$-lactamases (ESBL), AmpC $\beta$-lactamases
(AmpC) and metallo- $\beta$-lactamases (MBL). In a study from Brazil involving 84 ceftazidime resistant $P$. aeruginosa, $37 \%$ and $4 \%$ were found to produce MBL and ESBL respectively (16). In another study, $55 \%$ of the $P$. aeruginosa resistant to third generation cephalosporins were AmpC producers (14). However, there are only few studies from India, which have systematically studied the underlying mechanisms for ceftazidime resistance among $P$. aeruginosa isolates.

Therefore, this prospective study was conducted over a period of one year (January 2008 to December 2008) to determine the prevalence of ceftazidime resistance in $P$. aeruginosa and the rate of ESBL, AmpC and MBL production among the ceftazidime resistant $P$. aeruginosa isolated from the in-patients and the out-patients of a tertiary care hospital. A

*Corresponding Author. Mailing address: Department of Microbiology, Mahatma Gandhi Medical College and Research Institute, Pondicherry, India.; E-mail: drumadevis@yahoo.com 
total of $114 P$. aeruginosa isolated from both the in-patients and the out-patients were included in this study. They were isolated from various specimens such as pus, sputum, tracheal aspirate, blood and urine received in our lab during the study period. The isolates were identified as $P$. aeruginosa based on standard bacteriological techniques (11). All the isolates were screened for ceftazidime resistance by Kirby-Bauer disc diffusion method according to Clinical Laboratory Standards Institute (CLSI) guidelines (8). The ceftazidime resistant isolates were tested for ESBL, AmpC and MBL production. ESBL production was detected by double disc synergy test according to CLSI guidelines (9). Production of AmpC $\beta$-lactamases was detected by AmpC disc test as described earlier (19). MBL production was detected using EDTA disc synergy (EDS) test according to Yong et al (21). Susceptibility of the isolates to gentamicin, amikacin, ciprofloxacin, netilmicin, piperacillin/ tazobactam and imipenem were also tested using Kirby-Bauer disc diffusion method.

Of the 114 isolates of $P$. aeruginosa, 78 were isolated from in-patients, while the remaining 36 were obtained from outpatients. Ceftazidime resistance was observed among 51 of the 78 $(65 \%)$ isolates and 16 of the $36(44 \%)$ isolates from the in-patients and the out-patients respectively. Majority of the ceftazidime resistant $P$. aeruginosa were isolated from pus, while the remaining eight, seven, five and three isolates were from endotracheal aspirate, blood, sputum and urine respectively.

The rates of ESBL, AmpC and MBL production among the ceftazidime resistant Pseudomonas aeruginosa is summarised in Table 1. Four isolates of $P$. aeruginosa obtained from in-patients were positive for both ESBL and MBL. Another three isolates from in-patients were positive for both AmpC and MBL. Of the 44 MBL producers, 26 were detected by both meropenem and ceftazidime in EDS, while an additional 14 were detected only by EDTA-ceftazidime combination and another 4 were detected only by EDTA-meropenem combination. The antibiotic sensitivity patterns of the ESBL and MBL producing P. aeruginosa are summarized in Table 2.

Table 1. Proportion of ESBL, AmpC and MBL producers among the ceftazidime resistant Pseudomonas aeruginosa

\begin{tabular}{lcccc}
\hline & $\begin{array}{c}\text { No. of ceftazidime } \\
\text { resistant isolates }\end{array}$ & $\begin{array}{c}\text { ESBL Positive } \\
(\mathbf{\%})\end{array}$ & $\begin{array}{c}\text { AmpC Positive } \\
\mathbf{( \% )}\end{array}$ & $\begin{array}{c}\text { MBL Positive } \\
(\mathbf{\%})\end{array}$ \\
\hline In-patients & 51 & $11(21.6)$ & $6(11.8)$ & $38(74.5)$ \\
Out-patients & 16 & $2(12.5)$ & $5(31.3)$ & $6(37.5)$ \\
Total & 67 & $13(19.4)$ & $11(16.4)$ & $44(65.7)$ \\
\hline
\end{tabular}

ESBL - Extended spectrum $\beta$-lactamase

MBL - Metallo- $\beta$-lactamase

$\mathrm{AmpC}-\mathrm{AmpC} \beta$-lactamase

Table 2. Antibiotic sensitivity pattern of ESBL and MBL producing Pseudomonas aeruginosa

\begin{tabular}{|c|c|c|c|c|c|c|}
\hline & & & No. of sus & le isolates & & \\
\hline & GEN & AMK & CIP & NET & PTZ & IPM \\
\hline $\begin{array}{l}\text { ESBL } \\
\text { producers } \\
(\mathrm{n}=13)\end{array}$ & $4(30.8)$ & $7(53.8)$ & $6(46.2)$ & $7(53.8)$ & $9(69.2)$ & $13(100)$ \\
\hline $\begin{array}{l}\text { MBL } \\
\text { producers } \\
(\mathrm{n}=44)\end{array}$ & $8(18.2)$ & $12(27.3)$ & $6(13.6)$ & $14(31.8)$ & $15(34.1)$ & $2(4.5)$ \\
\hline
\end{tabular}


Ceftazidime is a third generation cephalosporin used frequently for the treatment of infections caused by $P$. aeruginosa. However, the resistance to ceftazidime is increasing at an alarming rate, complicating the clinical management of patients infected with such isolates. In this study, a high level of resistance to ceftazidime was observed among the $P$. aeruginosa isolates. The ceftazidime resistance was relatively more among the isolates from the in-patients compared to those from out-patients. Similarly, in a study from Iran, $73.4 \%$ of $P$. aeruginosa were observed to be resistant to ceftazidime (18). In another study from India, $78.4 \%$ of $P$. aeruginosa isolates displayed resistance to third generation cephalosporins (1).

Ceftazidime resistance is mainly mediated by production of $\beta$-lactamases such as ESBL, MBL and occasionally AmpC$\beta$-lactamases (17). Besides production of various $\beta$-lactamases, other mechanisms such as the lack of drug penetration due to mutation in porins, loss of certain outer membrane proteins and efflux pumps can also contribute for resistance to $\beta$-lactams (13). Extended-spectrum $\beta$-lactamases are enzymes that mediate resistance to extended-spectrum cephalosporins and monobactams (5). In the present study we observed that $19.4 \%$ of the ceftazidime-resistant isolates were ESBL producers. Likewise, in two other studies from India and Brazil similar high rates of ESBL production of about $21 \%$ was observed among the ceftazidime-resistant $P$. aeruginosa isolates $(1,17)$. These observations suggest that the ESBLs which are generally widespread among members of Enterobacteriaceae are also increasingly found in $P$. aeruginosa. Horizontal gene spread is considered to be responsible for the high frequency of ESBLs detected in $P$. aeruginosa (12). In our study about $16.4 \%$ of the ceftazidime-resistant isolates were noted to produce AmpC- $\beta$ lactamases. However, in a similar study from India, AmpC production was observed in $55.5 \%$ of the ceftazidime resistant Pseudomonas spp (14). Therefore, AmpC production is also an important mechanism for ceftazidime resistance among $P$. aeruginosa.
Metallo- $\beta$-lactamases, which require divalent cations, usually zinc, as metal cofactors for enzyme activity are very broad spectrum $\beta$-lactamases with ability to hydrolyze virtually all classes of $\beta$-lactams including extended-spectrum cephalosporins and carbapenems (20). EDTA disk synergy test using EDTA-ceftazidime and EDTA-meropenem combinations is commonly done for detection of MBL. In our study both the combinations simultaneously detected $26 \mathrm{MBL}$ producers. In addition, the EDTA-ceftazidime combination detected $14 \mathrm{MBL}$ producers, which were missed by the EDTA-meropenem combination. This increased sensitivity of EDTA-ceftazidime combination was also noted in two other studies from India and Japan $(3,13)$. The better sensitivity of ceftazidime could be attributed to its ability to produce a marked inhibitory effect with EDTA (13). However, four MBL producers were identified only by EDTA-meropenem combination and not by EDTA-ceftazidime combination. These four isolates were also found to be ESBL producers. Therefore, it is suggested that in these isolates the synergistic zone of inhibition between EDTA and ceftazidime was probably masked by the resistance to ceftazidime conferred by the ESBL, which is independent of zinc ions for its action. Thus, both EDTA-meropenem and EDTA-ceftazidime combination must be used simultaneously to accurately detect all the MBL producers.

In an earlier study from India only $8 \%$ strains were observed to be MBL producers (2). However, in our study $74.5 \%$ of the ceftazidime-resistant $P$. aeruginosa isolates from the in-patients were found to be MBL producers. Similarly in a study from Iran, $53 \%$ of the 94 ceftazidime-resistant $P$. aeruginosa isolates were noted to be MBL producers (18). In another recent study performed in a Brazilian teaching hospital involving 43 ceftazidime-resistant $P$. aeruginosa isolates from patients with bloodstream infections, $70 \%$ were found to be MBL producers (17). These findings clearly suggest that rate of MBL production is increasing among the $P$. aeruginosa isolates and it could be an important underlying mechanism for the ceftazidime resistance. The emergence of MBL producing 
multidrug-resistant $P$. aeruginosa presents a very serious problem for the control of clinical infections (10). The recommended treatment for MBL producing $P$. aeruginosa is a combination of colistin and rifampicin (with or without tigecycline) (13).

In our study we observed that the ESBL and MBL producing $P$. aeruginosa were often resistant to other classes of antibiotics such as aminoglycosides and fluoroquinolones. This could be due to co-existence of genes encoding drug resistance to those antibiotics on the plasmids carrying ESBL and MBL genes (1). Fused gene cassettes carrying MBL gene and an aacA4 gene that encodes aminoglycoside resistance are also known to exist (20).

In the present study four isolates of $P$. aeruginosa were positive for both ESBL and MBL. Such combinations of $\beta$ lactamases in a single strain have been recently reported in studies from Brazil, Italy and Argentina $(15,16)$. Based on molecular methods these studies have shown co-existence of PER-1 (ESBL) with VIM-2 (MBL) and GES-1 (ESBL) with VIM-11 (MBL) (15,16). The emergence of $P$. aeruginosa possessing both these enzymes is a major public health concern necessitating efficient detection and intervention to control drug resistance.

The major limitation of this study is our failure to perform molecular techniques to detect the various ESBL, AmpC and MBL genes in our isolates. The other limitation is that we have not looked for the other possible resistance mechanisms such as efflux pump, loss of OprD, etc which also could have been responsible for the ceftazidime resistance in our isolates.

To conclude, in this study a high level of resistance to ceftazidime was observed among $P$. aeruginosa, especially in the isolates from in-patients. Based on phenotypic tests, a very high rate of MBL, ESBL and AmpC production was observed among the ceftazidime resistant $P$. aeruginosa. The relatively increased production of MBL compared to ESBL or AmpC in our isolates suggests that MBL production is an important contributing factor for ceftazidime resistance among $P$. aeruginosa. The emergence of $P$. aeruginosa possessing combinations of $\beta$-lactamases is a major public health concern necessitating efficient detection and intervention to control drug resistance.

\section{REFERENCES}

1. Aggarwal, R.; Chaudhary, U.; Bala, K. (2008). Detection of extendedspectrum beta-lactamase in Pseudomonas aeruginosa. Indian J Pathol Microbiol. 51, 222-224.

2. Agrawal, G.; Lodhi, R.B.; Kamalakar, U.P.; Khadse, R.K.; Jalgaonkar, S.V. (2008). Study of metallo-beta-lactamase production in clinical isolates of Pseudomonas aeruginosa. Indian J Med Microbiol. 26, 349351.

3. Arakawa, Y.; Shibata, N.; Shibayama, K.; Kurokawa, H.; Yagi, T.; Fujiwara, H.; Goto, M. (2000). Convenient test for screening metallo-âlactamase-producing Gram-negative bacteria by using thiol compounds. J Clin Microbiol. 38, 40-43.

4. Bhattacharjee, A.; Anupurba, S.; Gaur, A.; Sen, M.R. (2008). Prevalence of inducible AmpC beta-lactamase-producing Pseudomonas aeruginosa in a tertiary care hospital in northern India. Indian J Med Microbiol. 26, 89-90.

5. Bradford, P.A. (2001). Extended-spectrum beta-lactamases in the $21 \mathrm{st}$ century: characterization, epidemiology, and detection of this important resistance threat. Clin Microbiol Rev. 14, 933-51.

6. Chastre, J.; Fagon, J.Y. (2002). Ventilator-associated pneumonia. Am J Respir Crit Care Med. 165, 867-903.

7. Chen, H.Y.; Yuan, M.; Livermore, D.M. (1995). Mechanisms of resistance to beta-lactam antibiotics amongst Pseudomonas aeruginosa isolates collected in the UK in 1993. J Med Microbiol. 43, 300-309.

8. Clinical Laboratory Standards Institute (2006). Performance standards for antimicrobial disk susceptibility tests. $9^{\text {th }}$ ed. Approved standards, M2-A9, Wayne, PA.

9. Clinical Laboratory Standards Institute (2007). Performance standards for antimicrobial susceptibility testing. Sixteenth informational supplement. Approved standards, M100 -S16, Wayne, PA.

10. Dong, F.; Xu, X.W.; Song, W.Q.; Lu, P.; Yu, S.J.; Yang, Y.H.; Shen, X.Z. (2008). Characterization of multidrug-resistant and metallo-betalactamase-producing Pseudomonas aeruginosa isolates from a paediatric clinic in China. Chin Med J (Engl). 121, 1611-1616.

11. Govan, J.R.V. (1996). Pseudomonas, Stenotrophomonas, Burkholderia. In: Collee, J.G., Fraser, A.C., Marmion, B.P., Simmons, A.(eds). Mackie \& McCartney Practical Medical Microbiology. Churchill Livingstone, New York, USA, p.413-424.

12. Jiang, X.; Zhang, Z.; Li, M.; Zhou, D.; Ruan, F.; Lu, Y. (2006). 
Detection of extended-spectrum beta-lactamases in clinical isolates of Pseudomonas aeruginosa. Antimicrob Agents Chemother. 50, 2990-2995.

13. Noyal, M.J.; Menezes, G.A.; Harish, B.N.; Sujatha, S.; Parija, S.C. (2009). Simple screening tests for detection of carbapenemases in clinical isolates of nonfermentative Gram-negative bacteria. Indian $J$ Med Res. 129, 707-712.

14. Parveen, M.; Harish, B. N.; Parija, S. C. (2010). AmpC beta lactamases among gram negative clinical isolates from a tertiary hospital, South India. Braz J Microbiol. 41, 596-602.

15. Pasteran, F.; Faccone, D.; Petroni, A.; Rapoport, M.; Galas, M.; Vazquez, M.; Procopio, A. (2005). Novel variant (bla(VIM-11)) of the metallo-\{beta\}-lactamase bla(VIM) family in a GES-1 extendedspectrum-\{beta\}-lactamase-producing Pseudomonas aeruginosa clinical isolate in Argentina. Antimicrob Agents Chemother. 49, 474-475.

16. Pellegrino, F.L.; Teixeira, L.M.; Carvalho Md, M.G.; Aranha, N.S., Pinto De, O.M., Mello Sampaio, J.L., D'Avila, F.A., Ferreira, A.L., morim Ed, E.L., Riley, L.W., Moreira, B.M. (2002). Occurrence of a multidrugresistant Pseudomonas aeruginosa clone in different hospitals in Rio de Janeiro, Brazil. J Clin Microbiol. 40, 2420-2424.
17. Picao, R.C.; Poirel, L.; Gales, A.C.; Nordmann, P. (2009). Diversity of beta-lactamases produced by ceftazidime-resistant Pseudomonas aeruginosa isolates causing bloodstream infections in Brazil. Antimicrob Agents Chemother. 53, 3908-3913.

18. Saderi, H.; Karimi, Z.; Owlia, P.; Bahar, M.A.; Rad, S.M.B.A. (2008). Phenotypic Detection of Metallo-beta-lactamase Producing Pseudomonas aeruginosa Strains Isolated from Burned Patients. Iranian Journal of Pathology. 3, 20-24.

19. Singhal, S.; Mathur, T.; Khan, S.; Upadhyay, D. J.; Chugh, S.; Gaind, R.; Rattan, A. (2005). Evaluation of methods for AmpC beta-lactamase in gram negative clinical isolates from tertiary care hospitals. Indian $\mathrm{J} \mathrm{Med}$ Microbiol. 23, 120-124.

20. Walsh, T.R.; Toleman, M.A.; Poirel, L.; Nordmann, P. (2005). Metallobeta-lactamases: the quiet before the storm? Clin Microbiol Rev. 18, 306325 .

21. Yong, D.; Lee, K.; Yum, J.H.; Shin, H.B.; Rossolini, G.M.; Chong, Y. (2002). Imipenem-EDTA disk method for differentiation of metallo-betalactamase-producing clinical isolates of Pseudomonas spp. and Acinetobacter spp. J Clin Microbiol. 40, 3798-3801. 